\title{
Is Elevated First Trimester Mean Arterial Blood Pressure Associated With Increased Risk of Placenta Accreta?
}

\section{Fengge Wang}

Jining Medical University

Peng Lin

Jining Medical University

Liangxi Zhu

Jining Medical University

Miaomiao Qu

Jining Medical University

Fangxiang Dong

Jining Medical University

Hua Shu

Jining Medical University

Xueqin Feng

Jining Medical University

Miao Liu

Jining Medical University

Chunlong Su

Jining Medical University

Tiantian Yu

Jining Medical University

Bin Zhang

Jining Medical University

Dongmei Man ( $\nabla$ mandongmei@163.com )

Jining medical university

\section{Research}

Keywords: mean arterial blood pressure, first trimester, placenta accreta, risk factors, association

Posted Date: May 26th, 2021

DOl: https://doi.org/10.21203/rs.3.rs-547386/v1 
License: (c) (i) This work is licensed under a Creative Commons Attribution 4.0 International License. Read Full License 


\section{Abstract}

Purpose: The present study investigated whether first trimester mean arterial blood pressure (MAP) differed among pregnancies with placenta accreta and healthy pregnancies.

Methods: We recruited 176 pregnant females totally from 1 January 2016 to 30 September 2018 in this study, as follows: 65 cases of placenta accreta and 111 cases of BMI and age matched, healthy pregnant controls. First trimester mean arterial blood pressure (MAP) were acquired from laboratory data files. Multiple logistic regression analysis were used to study analyzed the probable risk predictor of placenta accreta.

Results: The performance of MAP was lower in healthy pregnancies. The MAP of the placenta accreta group was significantly higher than that of the cont rol group $(p=0.001<0.05)$. Our results also showed that MAP was significantly positively associated with placenta accreta after adjusting for age, BMI, fertilization type, gestational week at time of blood pressure measurement, and previous cesarean section history (odds ratio [ $\beta]$ : $1.11 ; 95 \%$ confidence interval $[C]$ ]: $1.04-1.69 ; p=0.0013<0.05$ ). In addition, smoking during pregnancy ( $\beta: 7.57 ; 95 \% \mathrm{Cl}: 1.41-40.72 ; p=0.018<0.05)$ and previous cesarean section history ( $\beta$ : $2.57 ; 95 \% \mathrm{Cl}: 1.19-5.54 ; p=0.016<0.05)$ were significantly positively associated with placenta accreta.

Conclusions: Increased first trimester MAP was significantly positively associated with placenta accreta, suggesting the potential role of MAP in identifying high-risk pregnancies for placenta accreta. Smoking during pregnancy and previous cesarean section history may be risk factors for placenta accreta.

\section{Background}

In the field of obstetrics, placenta accreta is a life-threating complication that can lead to serious bleeding, secondary infection, uterine perforation, shock, and even death [1]. Placenta accreta affects approximately 3 out of 1000 pregnancies [2, 3]. In addition, the incidence of this condition is rising [4]. The prevalence of placenta accreta continues to rise internationally, placing a significant impact on health resources. Placenta accreta occurs by 10 weeks, and accreta cases have been diagnosed as early as the first trimester [5]. Accurate antenatal diagnosis and prediction of placenta accreta are pivotal and important considering the gradually rising incidence and severe adverse outcomes of placenta accreta.

Evidence shows that accurate antenatal diagnosis of placenta accreta may reduce maternal peripartum hemorrhage and morbidity [6]. Ultrasound techniques such as ultrasound and magnetic resonance imaging (MRI) are mainly used in the current prenatal diagnosis of placenta accreta. However, the accuracy of MRI and ultrasound diagnosis of placenta accreta is controversial. In addition, many patients may not have access to a high-risk prenatal diagnosis center for antenatal examination [7-9]. The early accurate risk assessment of placenta accreta would identify high-risk women for targeted early prevention. In addition, the early accurate antenatal diagnosis of the risk of placenta accreta may allow for the improvement of the outcome by increasing patient surveillance or by initiating therapeutic 
interventions. Thus, developing an effective and convenient prediction strategy of placenta accreta at the first trimester is important to avoid adverse outcomes.

Extravillous trophoblast cells invade into the maternal decidua and play a critical role in the maintenance of a successful pregnancy [10]. Pre-eclampsia and gestational diabetes mellitus are pregnancyassociated diseases related to the invasion ability of trophoblasts [11-13]. By contrast, excessive trophoblast invasion, abnormal vascular remodeling, and decidual deficiency are the main pathophysiological mechanisms of placenta accreta [14]. These pregnancy-associated diseases are related to abnormal early trophoblastic invasion. First trimester mean arterial blood pressure (MAP) is closely related to some adverse pregnancy outcomes such as hypertensive disorders, pre-eclampsia, small for gestational age, and gestational diabetes mellitus [15-18]. In addition, first trimester MAP is typically used for assessing the risk of pre-eclampsia, small for gestational age, intrauterine growth restriction, and gestational diabetes mellitus during the trimesters of pregnancy [16-21]. The relationship between first trimester MAP and placenta accreta is still unclear. Thus, the present study aimed to determine the relationship between first trimester MAP and placenta accreta. First trimester MAP may help to improve the prenatal diagnosis of placenta accreta. In addition, knowing the risks of placenta accreta early may contribute to the accuracy of interpretation of MRI or sonogram findings and the customization of personalized diagnosis and treatment schemes.

\section{Methods}

\section{Study settings and population}

We conducted a retrospective study in the Department of Obstetrics of the Affiliated Hospital of Jining Medical University between 1 January 2016 and 30 January 2020. A total of 215 pregnancies were involved in our retrospective study prior to being screened according to the exclusion criteria. The inclusion criteria were as follows: (1) placenta accreta group: pregnant women were diagnosed as placenta accreta later histologically; (2) control group: age- and BMI-matched pregnant controls (Fig. 1). The exclusion criteria were as follows: (1) pregnant women whose delivery or clinical data were missing; (2) twin or multiple pregnancies and miscarriage or stillbirths; (3) pregnant women with trophoblast tumor, gestational diabetes, acute and chronic infectious diseases, and other pregnancy complications. Finally, a total of 176 cases were eligible in this study ( 111 for the control group and 65 for the placenta accreta group). Gestational age was compiled from last menstrual period (LMP) and confirmed by first trimester ultrasound where LMP was not known. The MAPs of all pregnant women in the study were measured and calculated during the first trimester according to the requirements of their attending doctors. Before systolic and diastolic blood pressure measurement, all pregnant women were required to rest for $15 \mathrm{~min}$. All pregnant women were in the sitting position with their arms supported at the level of the heart, and a small $(22 \mathrm{~cm})$, normal $(22-32 \mathrm{~cm})$, or large $(33-42 \mathrm{~cm})$ adult cuff was used, depending on the mid-arm circumference. Two recordings of blood pressure were made in both arms simultaneously. We calculated the final MAP as the average of all four measurements.[16] Additionally, maternal demographic characteristics, obstetrical and medical history, and other maternal information 
were collected from the maternity records of the Affiliated Hospital of Jining Medical University or the general medical practitioners of the women. To protect patient privacy, our report did not include participants' identifiable data. The study was approved by the Human Ethics Committee of the Affiliated Hospital of Jining Medical University (Shandong, China).

\section{Statistical analysis}

Based on whether the continuous variables are normally distributed, we presented continuous variables in this study as medium (min-max, skewed distribution) or mean \pm standard deviation (normal distribution). Categorical variables were expressed in frequency or percentage. We used $\chi 2$ (categorical variables), oneway ANOVA (normal distribution), or Kruskal-Wallis $\mathrm{H}$ test (skewed distribution). All analyses were performed with SPSS 22.0 (IBM SPSS Statistics for Windows, Version 22.0. IBM Corp. Armonk, NY, USA). Categorical data are shown with $\mathrm{n}$ (number) and percentage (\%). Binary logistic regression with single and multi-categorical predictor was used to determine the possible risk factors for placenta accreta. The data were examined at the $95 \%$ confidence level, and a p-value of $<0.05$ was considered significant.

\section{Results}

\section{Baseline characteristics and first trimester MAP of the two groups}

We recruited 215 gestational women, among which 176 were eligible. Two groups were included as follows: 111 age- and BMI-matched healthy controls and 65 placenta accreta patients. Table 1 lists the demographic characteristics, laboratory features, clinical history, and first trimester MAP of all groups. No statistical difference was observed in terms of age, BMI, height, gestational week at time of blood pressure measurement, cesarean hysterectomy incidence at the time of delivery, and mean birth weight in both groups. The incidence of smoking during pregnancy and previous cesarean section history in the placenta accreta group were significantly higher than those in the control group $(p=0.018<0.05, p=$ $0.016<0.05)$ (Table 1$)$. Delivery pregnancy week in the placenta accreta group was significantly lower than that in the control group $(p<0.05$, Table 1$)$. Blood transfusion incidence and vaginal bleeding incidence in the placenta accreta group were significantly higher than those in the control group $(p<0.05$, $p<0.05$, Table 1 ). Delivery pregnancy week and neonatal weight in the placenta accreta group were significantly decreased than those in the control group $(p<0.05, p<0.05$, Table 1$)$. The normally distributed first trimester MAP is expressed as the median (min-max) in Table 1. The median first trimester MAP of placenta accreta cases was 89.74 , which was significantly higher than that in the control group $(84.79, \mathrm{p}=0.001<0.05)$. 
Table 1

Baseline characteristics of subjects

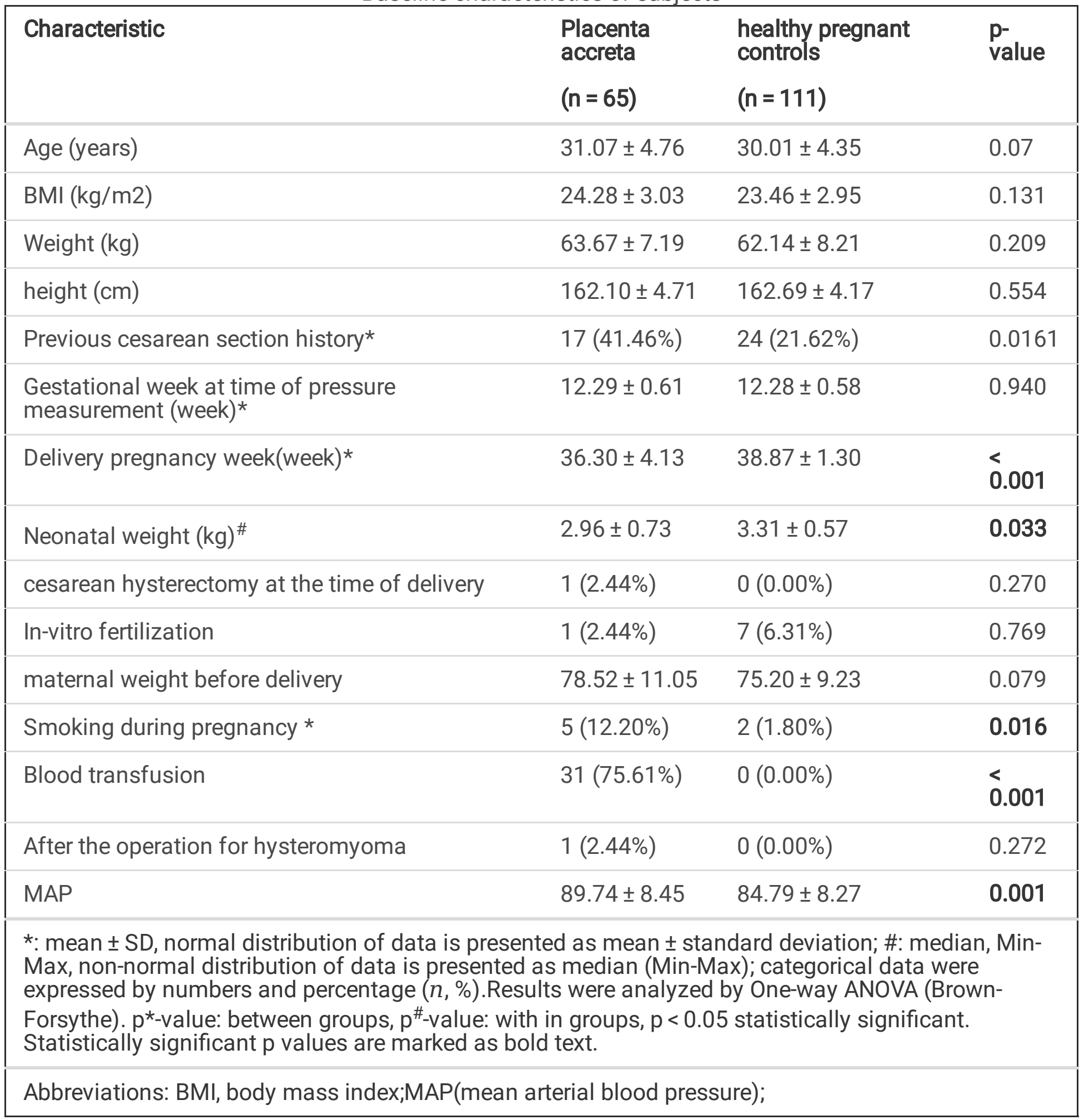

\section{Logistic regression analysis of the possible risk factors for placenta accreta}

Table 2 lists the median first trimester MAP and the results of univariate analyses of different parameters. Our results showed that age, BMl, height, gestational week at time of blood pressure measurement, invitro fertilization cesarean hysterectomy incidence at the time of delivery, and mean birth weight were not 
associated with placenta accreta $(p>0.05)$. Previous cesarean section history (odds ratio [ $\beta]: 2.57 ; 95 \%$ confidence interval $[\mathrm{Cl}]: 1.19-5.54 ; \mathrm{p}=0.016)$ and smoking during pregnancy $(\beta: 7.57 ; 95 \% \mathrm{Cl}: 1.41-$ $40.72 ; p=0.018)$ were positively associated with placenta accreta. Delivery pregnancy week $(\beta: 0.56 ; 95 \%$ Cl: $0.43-0.74 ; p<0.0001)$ and neonatal weight ( $\beta$ : $0.41 ; 95 \% \mathrm{Cl}: 0.23-0.75 ; p=0.004)$ were negatively associated with placenta accreta. In addition, our results showed that the value of first trimester MAP was significantly positively associated placenta accreta.

Table 2

Univariate analysis of different variables for placenta accreta

\begin{tabular}{|lll|}
\hline Covariate & $\beta(95 \% \mathrm{Cl})$ & $P$-value \\
\hline Age (years) & $1.08(0.99,1.17)$ & 0.0700 \\
\hline BMI & $1.10(0.97,1.23)$ & 0.1317 \\
\hline Weight $(\mathrm{kg})$ & $1.02(0.98,1.07)$ & 0.2911 \\
\hline Smoking during pregancy & $7.57(1.41,40.72)$ & $\mathbf{0 . 0 1 8 4}$ \\
\hline Previous cesarean section history & $2.57(1.19,5.54)$ & $\mathbf{0 . 0 1 6 1}$ \\
\hline Delivery pregnancy week (week) & $0.56(0.43,0.74)$ & $<0.0001$ \\
\hline In-vitro fertilization & $0.37(0.04,3.09)$ & 0.3567 \\
\hline Maternal weight before delivery & $1.04(1.00,1.07)$ & 0.0669 \\
\hline Neonatal weight (kg) & $0.41(0.23,0.75)$ & $\mathbf{0 . 0 0 4 0}$ \\
\hline MAP & $1.07(1.03,1.12)$ & $\mathbf{0 . 0 0 2 2}$ \\
\hline $\begin{array}{l}\text { *p< } 0.05 \text { statistically significant, NS; not significant, statistically significant p-values are marked as } \\
\text { bold text. }\end{array}$ & \\
\hline
\end{tabular}

Multivariate logistic regression analysis was further performed to evaluate the median first trimester MAP (Table 3). Table 3 lists the effect sizes ( $\beta$ ) and $95 \%$ Cls. In the unadjusted model, multivariate logistic regression analysis showed that elevated values of first trimester MAP were significantly positively associated with placenta accreta $(\beta: 1.07 ; 95 \% \mathrm{Cl}: 1.51-4.95 ; p=0.009<0.05)$. After adjusting for maternal age, BMI, smoking during pregnancy, gestational week at time of blood pressure measurement, and previous cesarean section history, elevated values of first trimester MAP remained significantly positively associated with placenta accreta ( $\beta$ : 1.11; $95 \% \mathrm{Cl}: 1.03-1.12 ; p=0.0022<0.05)$ (Table 3). Thus, increased values of first trimester MAP, previous cesarean section history, and smoking during pregnancy may be the most significant and positive risk factors related to the underlying mechanism of placenta accreta. 
Table 3

Relationship between MAP and placenta accreta in different models using multivariate linear regression

\begin{tabular}{|c|c|c|c|c|}
\hline \multirow[t]{2}{*}{ Variable } & \multicolumn{2}{|l|}{ Crude Model } & \multicolumn{2}{|l|}{ Adjusted } \\
\hline & $\beta(95 \% \mathrm{Cl})$ & P-value & $\beta(95 \% \mathrm{Cl})$ & $P$-vale \\
\hline \multirow[t]{2}{*}{ MAP } & 4.68 & 0.0008 & 4.83 & 0.0009 \\
\hline & $(1.90,11.54)$ & & $(1.91,12.24)$ & \\
\hline \multicolumn{5}{|c|}{$\begin{array}{l}\text { ** Logistic regression model (binary logistic regression with single- and multi-categorical predictors) } \\
\text { was used to determine the possible risk factors for placenta accreta. Adjusted: adjusted for maternal } \\
\text { age, BMl, and gestational week at time of blood sampling. }\end{array}$} \\
\hline
\end{tabular}

\section{Discussion}

MAP, a feasible tool and part of antenatal surveillance, has remained the target of scientific research in the prediction of pregnancy-related diseases $[17,22]$. The association between first trimester MAP and placenta accreta is still unclear. In our retrospective case-control study, we investigated the association between first trimester MAP and placenta accreta. Our results showed that elevated first trimester MAP remained significantly positively associated with placenta accreta. Furthermore, first trimester MAP, smoking during pregnancy, and previous cesarean section history were significantly positively associated with placenta accreta, indicating that they may be probable risk predictors of placenta accreta.

Placenta accreta refers to abnormal placental invasion to the uterine wall, which is characterized by invasion of trophoblasts into the myometrium, but not beyond $[23,24]$. Placenta accreta is a lifethreatening obstetrical disease and has a high maternal morbidity and mortality rate, presenting specific intrapartum challenges $[25,26]$. Placenta accreta affects about $2 \%$ of singleton deliveries, and its incidence is gradually increasing [27]. Considering the rising incidence and associated severe adverse outcomes of placenta accreta, methods that can accurately diagnose placenta accreta in the prenatal period before patients develop symptoms need to be developed so that plans for the prevention of bleeding and related complications and individualized treatment can be made. Currently, ultrasound and MRI are the methods used to diagnose placenta accreta. The diagnostic factors include the presence of placental lacunae, loss of the hypoechoic retroplacental interface, and hypervascularity of the interface between the placenta and the bladder or uterine wall. However, the effectiveness and accuracy of the above mentioned methods are controversial. Recently, an increasing number of studies showed that first trimester MAP is associated with adverse pregnancy outcomes $[28,29]$.

In the present study, we observed that elevated first trimester MAP remained significantly positively associated with placenta accreta. Previous studies showed that first trimester MAP values may change in pregnant women who have already developed or are destined to develop placenta accreta. First trimester 
MAP may be helpful to improve the antenatal diagnosis of placenta accreta. Furthermore, knowing the risks early may contribute to explain the MRI or sonogram findings more accurately.

MAP, the mean value of arterial pressure in one cardiac cycle, is a part of antenatal surveillance. In addition, higher levels of MAP are related to the increased risk of developing hypertension during pregnancy, pre-eclampsia, and gestational diabetes $[15,16,19]$. All these pregnant outcomes and placenta accreta belong to the disorders of placental development. Placenta accreta starts to develop in the first trimester [10].

In women with pre-eclampsia, the values of uterine artery pulsatility index and mean arterial pressure are increased. Interestingly, our findings showed that first trimester MAP is significantly increased in the placenta accreta group compared with that in the healthy control group. However, all the values are within normal range. Uteroplacental vasculature circulation undergoes significant structural and functional modifications to ensure an adequate blood supply to the developing placenta and the fetus in the first trimester [30]. In early stages of pregnancy, trophoblasts invade the uterine spiral arteries, replacing muscular and endothelial cells of the arterial wall and transforming them in low resistance vessels with an increased blood flow [31]. As mentioned above, placenta accreta starts to develop in the first trimester [10]. Thus, the probable mechanisms underlying this phenomenon may be that increased MAP within normal limits may provide more blood supply to the placental circulation and other organs of placenta accreta patients [32].

Considering the excessive trophoblast invasion and abnormal vascular remodeling of placenta accreta patients, these patients need more blood supply. Hence, factoring first trimester MAP into the risk assessment could better identify patients who are at risk of developing placenta accreta, and placenta accreta patients would be subjected to closer and refined monitoring and treatment.

In addition, we found that the incidence of smoking during pregnancy was significantly positively associated with placenta accreta in our study, suggesting that smoking during pregnancy may be related to the occurrence and development of placenta accreta. A potential mechanism is that cigarette smoke increases placental adrenomedullin expression and aggravates trophoblast invasion via the adrenomedullin pathway [26]. Excessive invasion of trophoblast cells leads to the occurrence and development of placenta accreta eventually.

The incidence of placenta accreta is increasing every year [33]. There are parallels between the increasing number of caesarean section cases and the increasing incidence of placenta accreta [34, 35]. Evidence shows that women with a history of caesarean delivery have the highest risk of developing placenta accreta [36-38]. In the present study, we found a significant positive association between previous cesarean section history and the incidence of placenta accreta. Our results also echo those of previous studies, suggesting that previous cesarean section history may be an important factor of placenta accreta $[33,39,40]$. The probable mechanisms underlying this phenomenon may include abnormal angiogenesis, abnormal trophoblast differentiation, and oxygen tension in the uterine scar [41, 42]. 
Despite our findings, our study has several limitations. One limitation is the small number of cases, which possibly introduces selection bias. Another limitation is that all pregnant women were from China, which fully minimized the confounding effects of ethnic background. Whether our results can be extended to other ethnic groups remains to be confirmed.

\section{Conclusion}

Taken together, increased values of first trimester MAP were significantly positively related to placenta accreta, suggesting the potential role of first trimester MAP in identifying high-risk pregnancies for placenta accreta. Smoking during pregnancy and previous cesarean section history may increase the risk of placenta accreta. Future large-scale studies investigating the association between placenta accreta and first trimester MAP need to be conducted.

\section{Abbreviations}

MAP: mean arterial blood pressure

BMl: body mass index

\section{Declarations}

\section{Funding:}

This study was supported by the Key Research and Development Program of Jining Science (No.2020YXNS007) and the Research Fund for Academician Lin He New Medicine, China (No. JYHL2019FMS14).

\section{Competing interests:}

The authors declare that they have no competing interests.

\section{Ethics approval and consent to participate:}

The study was approved by the Human Ethics Committee of the Affiliated Hospital of Jining Medical University (Shandong, China)(2019-zr-016).

\section{Consent for publication:}

Not applicable

\section{Availability of data and materials:}

The datasets used and/or analysed during the current study are available from the corresponding author on reasonable request. 
Code availability:

Not applicable

\section{Authors' contributions:}

Fengge Wang, Bin Zhang, and Dongmei Man conceived and designed the study. Fengge Wang designed the study. Fengge Wang drafted the manuscript. Shuxiong Chen, Fengge Wang, Miaomiao Qu, Hua Shu, and Miao Liu performed the statistical analysis. Fangxiang Dong, Chunlong Su, Tiantian Yu, and Liangxi Zhu created the charts of the manuscript. All authors have read and approved the final version of the manuscript.

\section{Declaration of interests}

The authors declare that they have no known competing financial interests or personal relationships that could have appeared to influence the work reported in this paper.

The authors declare the following financial interests/personal relationships which may be considered as potential competing interests:

\section{References}

1. Su HW, Yi YC, Tseng JJ, Chen WC, Chen YF, Kung HF, Chou MM: Maternal outcome after conservative management of abnormally invasive placenta. Taiwanese journal of obstetrics \& gynecology 2017 , 56(3):353-357.

2. Morgan EA, Sidebottom A, Vacquier M, Wunderlich W, Loichinger M: The effect of placental location in cases of placenta accreta spectrum. American journal of obstetrics and gynecology 2019, 221(4):357 e351-357 e355.

3. Bartels HC, Postle JD, Downey P, Brennan DJ: Placenta Accreta Spectrum: A Review of Pathology, Molecular Biology, and Biomarkers. Disease markers 2018, 2018:1507674.

4. McNally L, Zhou Y, Robinson JF, Zhao G, Chen LM, Chen H, Kim MY, Kapidzic M, Gormley M, Hannibal $\mathrm{R}$ et al: Up-regulated cytotrophoblast DOCK4 contributes to over-invasion in placenta accreta spectrum. Proceedings of the National Academy of Sciences of the United States of America 2020, 117(27):15852-15861.

5. Silver RM: Abnormal Placentation: Placenta Previa, Vasa Previa, and Placenta Accreta. Obstetrics and gynecology 2015, 126(3):654-668.

6. Tikkanen M, Paavonen J, Loukovaara M, Stefanovic V: Antenatal diagnosis of placenta accreta leads to reduced blood loss. Acta obstetricia et gynecologica Scandinavica 2011, 90(10):1140-1146.

7. Comstock CH, Love JJ, Jr., Bronsteen RA, Lee W, Vettraino IM, Huang RR, Lorenz RP: Sonographic detection of placenta accreta in the second and third trimesters of pregnancy. American journal of obstetrics and gynecology 2004, 190(4):1135-1140. 
8. Brown BP, Meyers ML: Placental magnetic resonance imaging Part Il: placenta accreta spectrum. Pediatric radiology 2020, 50(2):275-284.

9. Publications Committee SfM-FM, Belfort MA: Placenta accreta. American journal of obstetrics and gynecology 2010, 203(5):430-439.

10. Pijnenborg R, Dixon G, Robertson WB, Brosens I: Trophoblastic invasion of human decidua from 8 to 18 weeks of pregnancy. Placenta 1980, 1(1):3-19.

11. Kapustin RV, Drobintseva AO, Alekseenkova EN, Onopriychuk AR, Arzhanova ON, Polyakova VO, Kvetnoy IM: Placental protein expression of kisspeptin-1 (KISS1) and the kisspeptin-1 receptor (KISS1R) in pregnancy complicated by diabetes mellitus or preeclampsia. Archives of gynecology and obstetrics 2020, 301(2):437-445.

12. Fisher SJ: Why is placentation abnormal in preeclampsia? American journal of obstetrics and gynecology 2015, 213(4 Suppl):S115-122.

13. Heidari Z, Mahmoudzadeh-Sagheb H, Narouei M, Sheibak N: Effects of gestational diabetes mellitus on stereological parameters and extravillous trophoblast cells of placenta compared to the control group. Journal of obstetrics and gynaecology : the journal of the Institute of Obstetrics and Gynaecology 2019, 39(7):928-933.

14. Jauniaux E, Collins S, Burton GJ: Placenta accreta spectrum: pathophysiology and evidence-based anatomy for prenatal ultrasound imaging. American journal of obstetrics and gynecology 2018, 218(1):75-87.

15. Nevalainen J, Korpimaki T, Kouru H, Sairanen M, Ryynanen M: Performance of first trimester biochemical markers and mean arterial pressure in prediction of early-onset preeclampsia. Metabolism: clinical and experimental 2017, 75:6-15.

16. Chaemsaithong P, Pooh RK, Zheng M, Ma R, Chaiyasit N, Tokunaka M, Shaw SW, Seshadri S, Choolani $\mathrm{M}$, Wataganara $\mathrm{T}$ et al: Prospective evaluation of screening performance of first-trimester prediction models for preterm preeclampsia in an Asian population. American journal of obstetrics and gynecology 2019, 221(6):650 e651-650 e616.

17. Sweeting AN, Wong J, Appelblom H, Ross GP, Kouru H, Williams PF, Sairanen M, Hyett JA: A Novel Early Pregnancy Risk Prediction Model for Gestational Diabetes Mellitus. Fetal diagnosis and therapy 2019, 45(2):76-84.

18. Prajapati SR, Maitra N: Prediction of preeclampsia by a combination of maternal history, uterine artery Doppler, and mean arterial pressure (a prospective study of $\mathbf{2 0 0}$ cases). Journal of obstetrics and gynaecology of India 2013, 63(1):32-36.

19. Sweeting AN, Wong J, Appelblom H, Ross GP, Kouru H, Williams PF, Sairanen M, Hyett JA: A first trimester prediction model for gestational diabetes utilizing aneuploidy and preeclampsia screening markers. The journal of maternal-fetal \& neonatal medicine : the official journal of the European Association of Perinatal Medicine, the Federation of Asia and Oceania Perinatal Societies, the International Society of Perinatal Obstet 2018, 31(16):2122-2130. 
20. Onwudiwe N, Yu CK, Poon LC, Spiliopoulos I, Nicolaides KH: Prediction of preeclampsia by a combination of maternal history, uterine artery Doppler and mean arterial pressure. Ultrasound in obstetrics \& gynecology : the official journal of the International Society of Ultrasound in Obstetrics and Gynecology 2008, 32(7):877-883.

21. Litwinska E, Litwinska M, Oszukowski P, Szaflik K, Kaczmarek P: Combined screening for early and late preeclampsia and intrauterine growth restriction by maternal history, uterine artery Doppler, mean arterial pressure and biochemical markers. Advances in clinical and experimental medicine : official organ Wroclaw Medical University 2017, 26(3):439-448.

22. Poon LC, Shennan A, Hyett JA, Kapur A, Hadar E, Divakar H, McAuliffe F, da Silva Costa F, von Dadelszen $\mathrm{P}, \mathrm{Mclntyre} \mathrm{HD}$ et al: The International Federation of Gynecology and Obstetrics (FIGO) initiative on preeclampsia: A pragmatic guide for first-trimester screening and prevention. International journal of gynaecology and obstetrics: the official organ of the International Federation of Gynaecology and Obstetrics 2019, 145 Suppl 1:1-33.

23. Di Mascio D, Cali G, D'Antonio F: Updates on the management of placenta accreta spectrum. Minerva ginecologica 2019, 71(2):113-120.

24. Jauniaux E, Jurkovic D: Placenta accreta: pathogenesis of a 20th century iatrogenic uterine disease. Placenta 2012, 33(4):244-251.

25. Fitzpatrick KE, Sellers S, Spark P, Kurinczuk JJ, Brocklehurst P, Knight M: Incidence and risk factors for placenta accreta/increta/percreta in the UK: a national case-control study. PloS one 2012, 7(12):e52893.

26. Collins SL, Alemdar B, van Beekhuizen HJ, Bertholdt C, Braun T, Calda P, Delorme P, Duvekot JJ, Gronbeck $\mathrm{L}$, Kayem $\mathrm{G}$ et al: Evidence-based guidelines for the management of abnormally invasive placenta: recommendations from the International Society for Abnormally Invasive Placenta. American journal of obstetrics and gynecology 2019, 220(6):511-526.

27. Dombrowski MP, Bottoms SF, Saleh AA, Hurd WW, Romero R: Third stage of labor: analysis of duration and clinical practice. American journal of obstetrics and gynecology 1995, 172(4 Pt 1):12791284.

28. Leijnse JEW, de Heus R, de Jager W, Rodenburg W, Peeters LLH, Franx A, Eijkelkamp N: First trimester placental vascularization and angiogenetic factors are associated with adverse pregnancy outcome. Pregnancy hypertension 2018, 13:87-94.

29. Krantz D, Goetzl L, Simpson JL, Thom E, Zachary J, Hallahan TW, Silver R, Pergament E, Platt LD, Filkins $\mathrm{K}$ et al: Association of extreme first-trimester free human chorionic gonadotropin-beta, pregnancy-associated plasma protein A, and nuchal translucency with intrauterine growth restriction and other adverse pregnancy outcomes. American journal of obstetrics and gynecology 2004, 191(4):1452-1458.

30. Oltra L, Reverte V, Garces B, Li Volti G, Moreno JM, Salazar FJ, Llinas MT: Trophoblast-induced spiral artery remodelling and uteroplacental haemodynamics in pregnant rats with increased blood pressure induced by heme oxygenase inhibition. Placenta 2020, 89:91-98. 
31. Pijnenborg R, Vercruysse L, Hanssens M: The uterine spiral arteries in human pregnancy: facts and controversies. Placenta 2006, 27(9-10):939-958.

32. Karimu AL, Burton GJ: Star volume estimates of the intervillous clefts in the human placenta: how changes in umbilical arterial pressure might influence the maternal placental circulation. Journal of developmental physiology 1993, 19(3):137-142.

33. Silver RM, Barbour KD: Placenta accreta spectrum: accreta, increta, and percreta. Obstetrics and gynecology clinics of North America 2015, 42(2):381-402.

34. Vyshka G, Capari N, Shaqiri E: Placenta increta causing hemoperitoneum in the 26th week of pregnancy: a case report. Journal of medical case reports 2010, 4:412.

35. Shellhaas CS, Gilbert S, Landon MB, Varner MW, Leveno KJ, Hauth JC, Spong CY, Caritis SN, Wapner RJ, Sorokin $Y$ et al: The frequency and complication rates of hysterectomy accompanying cesarean delivery. Obstetrics and gynecology 2009, 114(2 Pt 1):224-229.

36. Silver RM, Landon MB, Rouse DJ, Leveno KJ, Spong CY, Thom EA, Moawad AH, Caritis SN, Harper M, Wapner RJ et al: Maternal morbidity associated with multiple repeat cesarean deliveries. Obstetrics and gynecology 2006, 107(6):1226-1232.

37. Kingdom JC, Hobson SR, Murji A, Allen L, Windrim RC, Lockhart E, Collins SL, Soleymani Majd H, Alazzam M, Naaisa F et al: Minimizing surgical blood loss at cesarean hysterectomy for placenta previa with evidence of placenta increta or placenta percreta: the state of play in 2020. American journal of obstetrics and gynecology 2020.

38. Ruiz-Perez R, Delgado Lopez-Cozar E, Jimenez-Contreras E: [Principles and criteria used by the National Evaluation Committee of Research Activity (CNEAI-Spain) for the assessment of scientific publications: 1989-2009]. Psicothema 2010, 22(4):898-908.

39. Dreux S, Salomon LJ, Muller F, Goffinet F, Oury JF, Group ABAS, Sentilhes L: Second-trimester maternal serum markers and placenta accreta. Prenatal diagnosis 2012, 32(10):1010-1012.

40. Luo L, Sun Q, Ying D, Wu X, Yan P, Yang Y, Chen Z: Scoring system for the prediction of the severity of placenta accrete spectrum in women with placenta previa: a prospective observational study. Archives of gynecology and obstetrics 2019, 300(3):783-791.

41. Genbacev O, Zhou Y, Ludlow JW, Fisher SJ: Regulation of human placental development by oxygen tension. Science 1997, 277(5332):1669-1672.

42. Tantbirojn P, Crum CP, Parast MM: Pathophysiology of placenta creta: the role of decidua and extravillous trophoblast. Placenta 2008, 29(7):639-645.

\section{Figures}




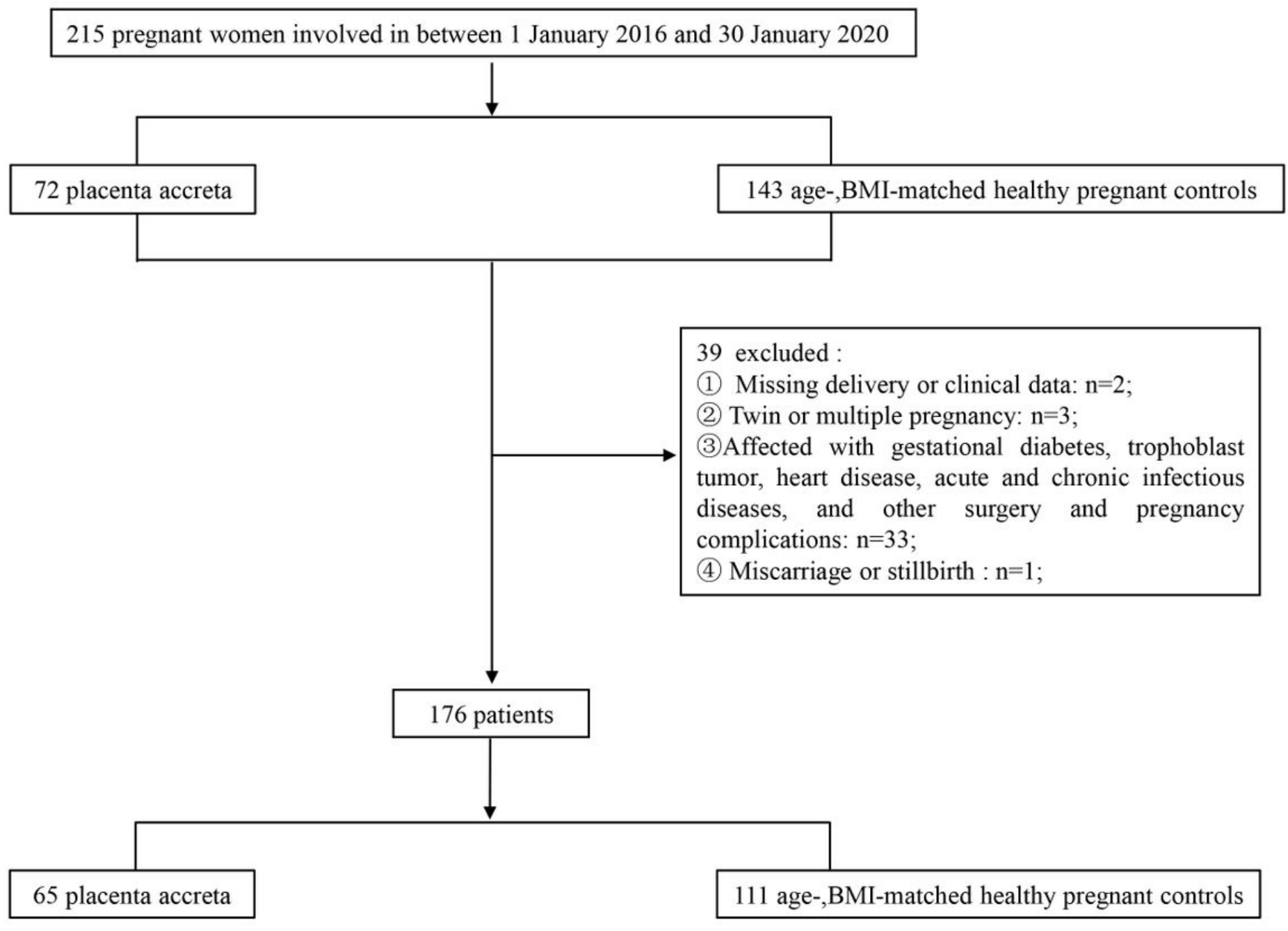

\section{Figure 1}

Flowchart of the study population In total, 176 patients from the Department of Obstetrics of the Affiliated Hospital of Jining Medical University, Shandong Province, China, between 1 January 2016 and 30 January 2020 were included in the study. Among them, 65 placenta accreta cases and 111 cases of age- and BMI-matched, healthy pregnant controls were further selected in accordance with the inclusion and exclusion criteria. 